\title{
Clinical utility of entecavir for chronic hepatitis B in Chinese patients
}

\author{
This article was published in the following Dove Press journal: \\ Drug Design, Development and Therapy \\ II December 2013 \\ Number of times this article has been viewed
}

\section{Jiyao Wang}

Zhongshan Hospital, Fudan University, Shanghai, Key Laboratory of Medical Molecular Virology of MOE/MOH, Shanghai, People's Republic of China
Correspondence: Jiyao Wang Zhongshan Hospital, Fudan University, I80\# Feng Lin RD, Shanghai 200032, People's Republic of China Tel +8621640419902117 Fax +862I 64432583 Email wang.jiyao@zs-hospital.sh.cn
Abstract: The People's Republic of China has one of the highest rates of hepatitis B virus (HBV) infection. This review summarizes recent data from studies of entecavir, one of the recommended first-line oral therapies for treating chronic hepatitis B, in Chinese HBVinfected patients. Long-term treatment with entecavir is efficacious and well tolerated, and studies comparing entecavir with other nucleos(t)ide therapies, such as lamivudine, adefovir, and telbivudine, demonstrate superior antiviral effects for entecavir therapy and comparable safety profiles. Entecavir monotherapy and combination treatment with other nucleos(t) ide analogs has been shown to be efficacious in the treatment of lamivudine-resistant and adefovir-resistant patients with HBV infection, as well as in patients with multidrug-resistant disease. Entecavir has also been shown to be effective in patients with $\mathrm{HBV}$-associated clinical morbidity, including cirrhosis and liver failure, as well as in preventing recurrence of HBV following liver transplantation and in preventing reactivation of HBV after immunosuppressive therapy. Although the cost of anti-HBV therapy is a particular concern in the People's Republic of China, a number of studies have recently demonstrated that entecavir (particularly long-term therapy) represents a more cost-effective treatment strategy compared with other nucleos(t)ide therapies. Further research is required to assess the effects of entecavir combination therapy on hepatitis B surface antigen clearance, and in drug-resistant patients in the People's Republic of China.

Keywords: hepatitis B, entecavir, People's Republic of China, nucleos(t)ides

\section{Introduction}

Hepatitis B virus (HBV) is a major cause of morbidity and mortality worldwide. The People's Republic of China has one of the world's highest rates of HBV infection despite the availability of an effective vaccine, ${ }^{1}$ and consequently chronic hepatitis B (CHB) remains a significant health burden in the People's Republic of China. It is estimated that 93 million individuals in the People's Republic of China are infected with HBV, ${ }^{1}$ including 20 million with active $\mathrm{CHB}^{2}$

The aims of this review are to describe the current status of anti-HBV therapy in the People's Republic of China, with particular focus on the use of entecavir, and to summarize the data describing the cost-effectiveness of entecavir compared with other anti-HBV therapies in Chinese patients with CHB. Data emerging from studies on the use of entecavir in special population of Chinese patients, including those with cirrhosis and those undergoing liver transplantation and those on immunosuppressive therapy, are also discussed. submit your manuscript | www.dovepress.com

Dovepress

http://dx.doi.org/10.2147/DDDT.S41423 


\section{Current status of anti-HBV treatment in the People's Republic of China}

In the updated 2010 Chinese CHB guidelines, two interferon (IFN)-based therapies (conventional IFN and pegylated-IFNalpha- $2 \mathrm{a}$ or pegylated-IFN-alpha- $2 \mathrm{~b}$ [pegIFN- $\alpha 2 \mathrm{a}$ or pegIFN$\alpha 2 \mathrm{~b}$, respectively]) and the nucleos(t)ides lamivudine, adefovir, telbivudine, and entecavir are currently recommended for the treatment of patients with hepatitis B e-antigen (HBeAg)positive $\mathrm{CHB} .{ }^{2}$ For patients with $\mathrm{HBeAg}$-negative $\mathrm{CHB}$ and those with cirrhosis, nucleos(t)ide analogs with high potency and low resistance rates, such as entecavir, are preferred. The Asian-Pacific consensus statement on the management of CHB recommends that conventional IFN or pegIFN- $\alpha 2 \mathrm{a}$, lamivudine, adefovir, entecavir, telbivudine, and the nucleotide analog, tenofovir, can all be considered for initial therapy in patients without liver decompensation, although tenofovir has not yet been launched in the People's Republic of China. ${ }^{3}$

A recent study investigating trends in the management of CHB at a teaching hospital in South West China demonstrated that although the majority of physicians surveyed were aware of the Chinese consensus recommendations for the management of CHB, $28 \%$ would recommend conventional IFN or pegIFN, but not nucleos(t)ide therapy, as the initial treatment for patients with hepatitis B surface antigen (HBsAg)-positive disease. ${ }^{4}$ Approximately $37 \%$ (44/120) of the physicians surveyed reported experience with entecavir, which was lower than the number of physicians who reported experience with lamivudine $(84 \% ; 101 / 120)$ or adefovir $(57 \% ; 68 / 120)$ nucleos $(\mathrm{t})$ ide therapy, thus demonstrating a lack of awareness of guidelines, particularly on the use of entecavir. Furthermore, the proportion of patients with $\mathrm{CHB}$ receiving any anti-HBV treatment in the People's Republic of China is often low, despite the wide availability of therapies. ${ }^{5}$ Although entecavir has been included in the government health insurance program since 2009, some patients still have to pay for their own treatment where costs exceed their annual limits, which means treatment remains unaffordable for many individuals. ${ }^{5}$

According to data released by the market research firm, IMS Health, entecavir currently accounts for $26 \%$ of the market share for HBV treatments in the People's Republic of China, compared with $39 \%$ for adefovir. ${ }^{6}$ Lamivudine and telbivudine, respectively, account for $22 \%$ and $8 \%$ of the market share.

Because the cost of therapy is an issue in the People's Republic of China, particularly for entecavir, many pharmaceutical companies, including Jiangsu Chia-Tai Tianqing Pharmaceutical Co., Ltd. (Jiangsu, People's Republic of China), Jiangxi Qingfeng Pharmaceutical Co., Ltd. (Jiangxi, People's Republic of China), and Dawnrays Pharmaceutical Holdings Ltd. (Hong Kong), produce generic versions of entecavir. However, many of these generic entecavir drugs are only supported by data from bioequivalence studies, and there is a distinct lack of long-term efficacy studies. In general, generic entecavir has been shown to be effective in the management of CHB, with the advantage of low cost and high cost-effectiveness compared with Baraclude $^{\circledR}$ (Bristol-Myers Squibb, New York, NY, USA). ${ }^{7-10}$ However, the majority of these studies are observational, and no investigations to date have demonstrated enhanced efficacy or an improved safety profile with generic entecavir compared with Baraclude. ${ }^{11}$ Therefore, although generic entecavir drugs are less expensive, their long-term efficacy and safety remains to be fully established.

\section{Efficacy and safety of entecavir in treatment-naïve Chinese patients with CHB}

A number of studies have demonstrated that entecavir is an effective and well tolerated treatment for Chinese patients with CHB. ${ }^{12,13}$ A retrospective real-life study assessed the efficacy and safety of long-term treatment with entecavir in 230 treatment-naïve Chinese patients with CHB treated at the Department of Infectious Diseases, The Third Affiliated Hospital, Sun Yat-sen University, between June 2006 and September 2012. ${ }^{12}$ Reductions in HBV DNA and increased virologic responses were observed at 12 and 24 weeks. Over the treatment period, incremental increases in rates of undetectable levels of HBV DNA were observed, ie, $>90 \%$ after 2 years and up to $100 \%$ after 5 years, demonstrating that entecavir is a potent anti-HBV therapy (Figure 1). In addition, entecavir was well tolerated, with no serious adverse events reported.

The efficacy of entecavir in terms of viral suppression, drug resistance, and safety have also been examined in a large cohort study of treatment-naïve patients with CHB and treated with entecavir at the Department of Medicine, Queen Mary Hospital, University of Hong Kong, between July 2005 and November 2007. ${ }^{13}$ Incremental increases in rates of undetectable $\mathrm{HBV}$ DNA, $\mathrm{HBeAg}$ seroconversion, and alanine transaminase (ALT) normalization were observed, reaching $92 \%, 44 \%$, and $90 \%$, respectively, at year 3. Overall, $100 \%$ and $77 \%$ of patients with baseline HBV DNA levels $<$ and $\geq 8 \log _{10}$ copies/mL, respectively, had undetectable HBV DNA at year 3. The cumulative rate of entecavir-resistant mutations was $1 \%$ at year 3 . Three patients experienced virologic breakthrough, comprising one patient who developed resistance, one patient with subsequent $\mathrm{HBeAg}$ seroconversion, and another patient with a 
A

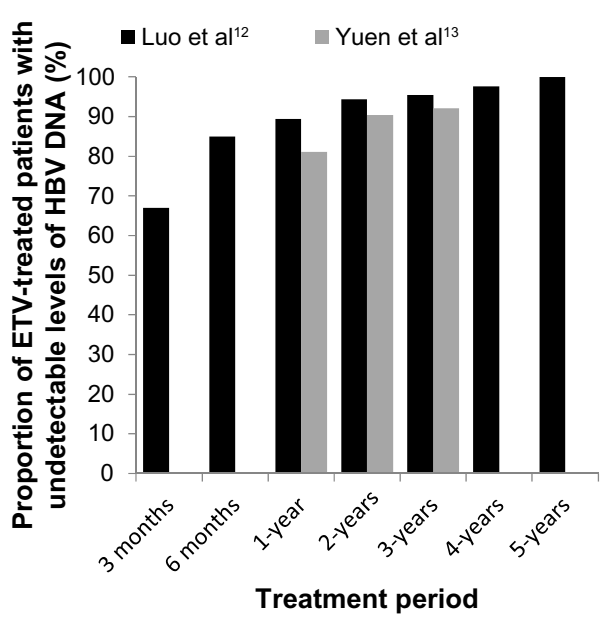

B

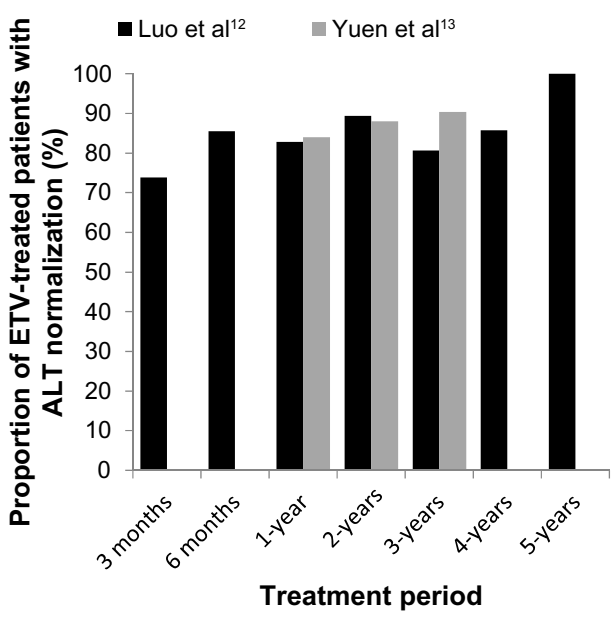

Figure I Effect of long-term ETV therapy on the proportion of patients with (A) undetectable HBV DNA and (B) ALT normalization.

Notes: Graph created using data taken from Luo et al, $2013^{12}$ and Yuen et al, $2011 .^{13}$ Data cannot be directly compared due to differences in experimental procedures between studies.

Abbreviations: ALT, alanine transaminase; DNA, deoxyribonucleic acid; HBV, hepatitis B virus; ETV, entecavir.

subsequent decline in HBV DNA levels. No serious adverse events were reported.

Taken together, these studies show that long-term therapy with entecavir is a highly effective anti-HBV treatment for Chinese patients, with a favorable tolerability profile. Indeed, in a retrospective study of 129 patients with CHB, although short-term treatment with entecavir rapidly suppressed HBV replication, it did not increase the short-term survival rate or prevent long-term progression to liver failure, thus supporting the use of long-term treatment strategies. ${ }^{14}$

Entecavir has also been demonstrated to be an effective and well tolerated treatment option for $\mathrm{HBV}$-infected patients with schistosomiasis, one of the world's most important helminth infections in terms of global burden of morbidity and mortality, particularly in the People's Republic of China. ${ }^{15}$ Patients with $\mathrm{CHB}$ and schistosomiasis typically suffer greater morbidity compared with those presenting with either infection alone. ${ }^{16}$ In a study assessing the efficacy and safety of entecavir in patients with CHB and schistosomiasis japonica, entecavir demonstrated statistically significant improvement in fibrosis markers after 52 weeks of treatment compared with patients who received no anti-HBV therapy. ${ }^{15}$ In addition, treatment with entecavir was associated with an improvement in Ishak fibrosis score. More than $80 \%$ of patients in the entecavir group achieved undetectable levels of HBV DNA, and almost 70\% achieved normalization of ALT. No serious adverse events were observed throughout the treatment period.

A randomized, double-blind, double-dummy, controlled, multicenter study compared the generic entecavir drug, TianDing (entecavir maleate tablets; Jiangsu Chia-Tai Tianqing
Pharmaceutical Co., Ltd.) with Baraclude in Chinese patients with $\mathrm{HBeAg}$-negative CHB. ${ }^{11}$ Similar efficacy and safety data were observed between the two treatment groups, with comparable decreases in mean HBV DNA levels, rates of undetectable HBV DNA at week 48, normalization of ALT, and incidence of adverse events. However, long-term studies are still required to establish the safety and efficacy of generic entecavir drugs in the People's Republic of China.

A novel estimation of the impact of entecavir on long-term mortality and morbidity rates for CHB in the People's Republic of China concluded that entecavir significantly slowed disease progression, thus reducing mortality rates. ${ }^{17}$ It is important to note, however, that hepatic steatosis is significantly associated with entecavir failure, with levels of HBV DNA clearance and seroconversion being markedly lowered. ${ }^{18}$

\section{Efficacy of entecavir compared with other HBV treatment options}

The Chinese guidelines for the treatment of CHB recommend use of therapies with high potency and low resistance, such as entecavir, for nucleos(t)ide-naïve patients. ${ }^{2} \mathrm{~A}$ review examining the evidence provided by one pharmacokinetic study and three clinical studies conducted in the People's Republic of China concluded that entecavir should be recommended as a first-line therapeutic choice among the currently available anti-HBV therapies. ${ }^{19}$ Overall, entecavir was associated with a rapid reduction in serum HBV DNA levels, with a reduction of $>5 \log _{10}$ copies $/ \mathrm{mL}$ by week 12 and undetectable levels in $75 \%$ of cases by week 48 . In addition, emergence of drug resistance in the nucleos $(\mathrm{t})$ ide-naïve patients was rare, demonstrating the potential efficacious nature of long-term treatment 
using entecavir. ${ }^{19}$ In support of this, entecavir has recently demonstrated superior antiviral efficacy compared with lamivudine, adefovir, and telbivudine. ${ }^{20}$ REALM (Randomized, Observational Study of Entecavir to Assess Long-term Outcomes Associated With Nucleoside/Nucleotide Monotherapy for Patients With Chronic HBV Infection) is an ongoing global, Phase IV, prospective, observational, open-label study comparing entecavir with other standard-of-care anti-HBV nucleos $(\mathrm{t})$ ide therapies in patients with CHB. The People's Republic of China substudy is investigating the long-term efficacy and safety of entecavir versus other standard-of-care therapies in patients enrolled at 50 centers in a real-world setting in the People's Republic of China. Interim results for the People's Republic of China substudy were presented at the 2013 Asian Pacific Association for the Study of the Liver conference, and demonstrated that more entecavir-treated patients $(n=1,724)$ achieved HBV DNA levels $<50 \mathrm{IU} / \mathrm{mL}$ at $24,48,96,144$, and 192 weeks compared with those receiving other standard-ofcare treatments (lamivudine $n=69$; adefovir $n=1,612$; telbivudine $n=39) .{ }^{20}$ Serious treatment-related adverse events were infrequent $(<1 \%)$ and comparable between the treatment arms. Overall, these results demonstrate superior antiviral efficacy of entecavir compared with lamivudine, adefovir, and telbivudine, and support the current Asian-Pacific treatment guidelines recommendation to use entecavir as a first-line therapeutic option for patients with CHB. In addition, a number of studies in the People's Republic of China have directly compared entecavir with other nucleos(t)ide analogs that are currently recommended, including lamivudine, adefovir, and telbivudine. ${ }^{21-29}$

\section{Entecavir versus lamivudine}

Entecavir has demonstrated efficacy superior to that of lamivudine in a number of studies comparing the two therapies in Chinese patients with CHB. ${ }^{21-24} \mathrm{~A}$ multicenter study evaluated the long-term efficacy of entecavir in patients who continued entecavir therapy for up to 144 weeks. ${ }^{21} \mathrm{~A}$ total of 519 patients received either entecavir or lamivudine in an initial 96-week randomized, double-blind, controlled efficacy study. Entecavir resulted in a greater reduction in serum HBV DNA levels from baseline at weeks 12 and 48 and a higher rate of undetectable HBV DNA compared with lamivudine. The authors concluded that entecavir was superior to lamivudine in achieving virologic and biological responses in Chinese patients with CHB.

A randomized, double-blind, controlled study assessed the safety and efficacy of entecavir versus lamivudine in HBeAg-positive, treatment-naïve patients from Jilin Province, the People's Republic of China. ${ }^{23}$ Rates of viral suppression at weeks 24 and 48 were significantly higher with entecavir than with lamivudine. The mean reduction in serum HBV DNA levels was also significantly greater in entecavir-treated patients. The safety profile was comparable between the two therapies.

Another study assessed the long-term efficacy and safety of entecavir and lamivudine in 519 nucleos(t)ide-naïve adults with CHB from 26 sites across the People's Republic of China over a follow-up period of 3 years. ${ }^{22}$ The mean reduction in HBV DNA levels at week 12 was significantly greater for the entecavir-treated group than for the lamivudine-treated group $\left(-5.07 \log _{10}\right.$ copies $/ \mathrm{mL}$ versus $-4.53 \log _{10}$ copies $/ \mathrm{mL}$; $P<0.001)$. Of 160 patients who received entecavir continuously for 144 weeks, $89 \%$ had undetectable serum HBV DNA levels, $86 \%$ showed normalization of ALT, $20 \%$ had loss of $\mathrm{HBeAg}, 8 \%$ experienced $\mathrm{HBeAg}$ seroconversion, and 3\% showed evidence of genotypic mutation.

Taken together, these studies demonstrate superior efficacy for entecavir compared with lamivudine in Chinese patients with CHB. However, in an earlier study comparing entecavir and lamivudine, entecavir demonstrated similar virologic, biochemical, and serologic outcomes in nucleos(t)ide-naïve Chinese patients with $\mathrm{CHB} .{ }^{24}$ Although a higher proportion of patients treated with entecavir achieved HBV DNA $<300$ copies $/ \mathrm{mL}$ ( $79 \%$ versus $46 \% ; P<0.0001$ ), similar proportions of entecavir-treated and lamivudine-treated patients achieved confirmed ALT normalization and HBeAg seroconversion, and the safety profile was similar between the groups.

\section{Entecavir versus adefovir}

To date, only one study has compared the efficacy of entecavir with that of adefovir in Chinese patients with CHB. This was a prospective study by Chen et al comparing the efficacy of adefovir with that of entecavir over a 2-year period in 100 treatment-naïve patients. ${ }^{25}$ The authors concluded that entecavir was significantly superior to adefovir, with entecavir therapy being associated with a greater mean reduction in HBV DNA levels compared with adefovir at 24 months $\left(-7.5 \log _{10}\right.$ copies $/ \mathrm{mL}$ versus $-6.3 \log _{10}$ copies $/ \mathrm{mL}$, respectively; $P=0.003)$. Both treatments demonstrated similar rates of ALT normalization, $\mathrm{HBeAg}$ seroconversion, and overall adverse events, and there were no instances of entecavir-associated or adefovir-associated mutations.

\section{Entecavir versus lamivudine plus adefovir}

A comparison of the efficacy of entecavir versus that of lamivudine and adefovir combination therapy in a prospective case-control study of Chinese patients with CHB 
demonstrated that both treatment strategies were equally effective in reducing HBV DNA levels in treatment-naïve patients. ${ }^{26}$ No statistically significant differences in mean reduction of HBV DNA levels or in rates of undetectable HBV DNA were observed between the two treatment groups over 48 weeks. The adverse event profiles for the two treatments were also comparable. However, another study demonstrated that entecavir had superior antiviral efficacy compared with combination therapy using lamivudine and adefovir. ${ }^{27}$ Fifty patients with $\mathrm{HBeAg}$-positive $\mathrm{CHB}$ were treated with entecavir or lamivudine plus adefovir over a treatment period of 96 weeks. Complete virologic response rates were significantly greater in the entecavir group compared with the lamivudine-adefovir combination therapy group (86\% versus $43 \%$; $P=0.003)$. Patients in both treatment groups demonstrated significant improvement in biochemical liver responses, and there were no cases of virologic breakthrough. Further, no treatment-related adverse events were reported throughout the treatment and follow-up periods.

\section{Entecavir versus telbivudine}

Entecavir has also demonstrated efficacy comparable with that of telbivudine. ${ }^{28}$ In a study of ethnic Han Chinese patients with previously untreated $\mathrm{HBeAg}$-positive $\mathrm{CHB}$, similar mean reductions in serum levels of HBV DNA at weeks 12 and 24 were observed with the two treatments, as well as similar proportions of patients achieving undetectable HBV DNA. Both treatments demonstrated comparable tolerability profiles. However, in a retrospective single-center study in patients from Taiwan, entecavir was associated with better clinical outcomes than was telbivudine. ${ }^{29}$ Rates of ALT normalization $(85 \%$ versus $78 \% ; P<0.048)$ and undetectable HBV DNA ( $96 \%$ versus $75 \% ; P<0.001$ ) were significantly higher for entecavir than for telbivudine, and the incidence of viral resistance $(1 \%$ versus $22 \% ; P<0.001)$ was significantly lower in the entecavir-treated group. HBeAg seroconversion and the cumulative incidence of hepatocellular carcinoma were comparable between the two treatment groups.

Taken together, these studies demonstrate that entecavir has a tolerability profile similar to that of the other nucleos $(\mathrm{t})$ ides, including adefovir and lamivudine, but is associated with superior antiviral efficacy in Chinese patients with CHB (Table 1).

\section{Cost-effectiveness of entecavir}

The cost of treatment plays an important role in the decision-making process when treating patients with $\mathrm{CHB}$ in the People's Republic of China because some patients are required to pay for their treatment. Although entecavir is widely available in the People's Republic of China, it is viewed as a more expensive treatment option compared with other nucleos(t)ide therapies. ${ }^{17}$ However, several studies have compared the overall cost-effectiveness of entecavir with that of other therapies and demonstrated that entecavir is a more cost-effective option. ${ }^{17,30,31}$ Wei et al used a Markov transition model of disease states based on available natural history data to simulate $\mathrm{CHB} .{ }^{17}$ Over a treatment duration of 5 years, entecavir was estimated to result in a cost-saving of US\$2.69 per day when compared with no treatment. In addition, longterm entecavir resulted in daily cost-savings of US\$2.33 and

Table I Summary of ETV efficacy versus other nucleos(t)ide therapies in Chinese patients with CHBa

\begin{tabular}{|c|c|c|c|c|c|c|c|c|}
\hline \multirow[t]{2}{*}{ Author } & \multicolumn{2}{|c|}{$\begin{array}{l}\text { HBV DNA } \\
<300 \text { copies/mL/ } \\
\text { undetectable HBV DNA }\end{array}$} & \multicolumn{2}{|c|}{$\begin{array}{l}\text { ALT normalization } \\
(\leq \mathrm{I} \times \text { ULN) }\end{array}$} & \multicolumn{2}{|c|}{ HBeAg loss } & \multicolumn{2}{|c|}{$\begin{array}{l}\mathrm{HBeAg} \\
\text { seroconversion }\end{array}$} \\
\hline & ETV & LVD & ETV & LVD & ETV & LVD & ETV & LVD \\
\hline Yao et $\left.a\right|^{21, b}$ & 79 & 46 & 96 & 92 & 27 & 27 & 21 & 23 \\
\hline Yao et $\mathrm{al}^{24, \mathrm{~b}}$ & 74 & 41 & 96 & 82 & 18 & 25 & II & 19 \\
\hline Yao et al ${ }^{19,22,37, c}$ & 76 & 43 & 90 & 78 & 18 & 20 & 15 & 18 \\
\hline \multirow[t]{2}{*}{ Ren et $\mathrm{al}^{23, \mathrm{c}}$} & $52^{d}$ & $36^{d}$ & 86 & 76 & NR & NR & 15 & 18 \\
\hline & ETV & ADV & ETV & ADV & ETV & ADV & ETV & ADV \\
\hline \multirow[t]{2}{*}{ Chen et $\mathrm{a}^{8,25,26,58, \mathrm{e}}$} & 84 & 55 & 97 & 88 & NR & NR & 25 & 24 \\
\hline & ETV & LVD + ADV & ETV & LVD + ADV & ETV & LVD + ADV & ETV & LVD + ADV \\
\hline \multirow[t]{2}{*}{ Wang et $\left.a\right|^{8,26,36, c}$} & $78^{d}$ & $8 I^{d}$ & 90 & 94 & 0 & 0 & 0 & 0 \\
\hline & ETV & LdT & ETV & LdT & ETV & LdT & ETV & LdT \\
\hline Tsai et $\mathrm{al}^{29, \mathrm{e}}$ & $97^{d}$ & $75^{d}$ & 85 & 78 & NR & NR & 43 & 46 \\
\hline Zheng et $\mathrm{al}^{28, \mathrm{f}}$ & $58^{d}$ & $68^{d}$ & 74 & 79 & 29 & 37 & 14 & 25 \\
\hline
\end{tabular}

Notes: All data are shown as percentage of patients, unless otherwise stated. ${ }^{\mathrm{a} D i r e c t}$ comparisons cannot be made between studies due to differences in treatment periods;

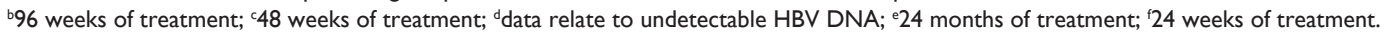

Abbreviations: ALT, alanine transaminase; CHB, chronic hepatitis B; DNA, deoxyribonucleic acid; HBeAg, hepatitis B e-antigen; HBV, hepatitis B virus; ULN, upper limit of normal; ETV, entecavir; LdT, tenofovir; LVD, lamivudine; ADV, adefovir. 
US\$1.73 at 1 year and 2 years, respectively, when compared with short-term use. Compared with other HBV therapies, entecavir offered daily cost-savings of US\$0.90-1.81. It was concluded that long-term therapy with entecavir is a favorable therapeutic and cost-effective strategy. In another study, a Markov analysis was conducted to examine the cost-effectiveness of nucleos(t)ide analog therapy for HBV in the People's Republic of China. ${ }^{30}$ In an HBeAg-positive cohort, treatment with entecavir was associated with a gain of 11.8 quality-adjusted life years, a $24 \%$ incidence of cirrhosis, a $15 \%$ incidence of hepatocellular carcinoma, and a $23 \%$ incidence of death. This study demonstrated that entecavir was the most cost-effective treatment option when compared with lamivudine and adefovir, and achieved the best health outcomes overall compared with other treatment options in the People's Republic of China.

In another economic analysis comparing entecavir and lamivudine for the treatment of CHB in Hong Kong, entecavir was expected to reduce the incidence of compensated cirrhosis, decompensated cirrhosis, and hepatocellular carcinoma by $42 \%, 57 \%$, and $49 \%$, respectively. ${ }^{31}$ Although the overall disease management cost for entecavir was $68 \%$ higher than that predicted for lamivudine for a treatment period of 2 years, this was reduced to $17 \%$ after projecting the 2-year treatment duration to 10 years. The incremental cost per quality-adjusted life year gained for entecavir compared with lamivudine was \$US13,759. As such, entecavir was considered to be cost-effective compared with lamivudine in treatment-naïve patients with CHB in Hong Kong, when long-term medical consequences were taken into consideration.

These studies demonstrate that although entecavir is associated with higher costs at the start of treatment, longterm therapy offers economic benefits for the treatment of CHB in Chinese patients when compared with other available nucleos(t)ide therapies.

\section{Entecavir in Chinese patients with drug-resistant CHB}

Lamivudine is associated with a high rate of viral resistance, ${ }^{32-35}$ and continued lamivudine treatment in lamivudine-resistant patients can result in increased viral load, hepatic flares that may lead to decompensation, or reversal of histologic improvement. In contrast, entecavir has a greater barrier to resistance in Chinese patients with CHB compared with lamivudine and adefovir. ${ }^{36}$ Thus, as well as having superior antiviral efficacy, entecavir is also associated with less resistance compared with other nucleos $(\mathrm{t})$ ide therapies in Chinese patients with $\mathrm{CHB}$.

\section{Entecavir in lamivudine-resistant CHB}

Although entecavir monotherapy has been shown to induce a marked reduction in HBV DNA levels in patients with lamivudine-refractory $\mathrm{CHB},{ }^{37}$ sequential use of nucleos $(\mathrm{t})$ ide monotherapies is associated with multidrug resistance, so add-on therapy may be a more effective therapeutic strategy in these patients. Indeed, a retrospective study concluded that while neither entecavir nor adefovir are optimal as monotherapies, switching to adefovir or entecavir combination therapy may be the most effective treatment regimen in lamivudine-resistant patients with CHB. ${ }^{38}$ The benefits of combination therapy using entecavir and adefovir were further demonstrated in a study conducted in 91 adult patients with lamivudine-resistant CHB (Table 2A). ${ }^{39}$ This was a comparative study of adefovir monotherapy $(n=29)$, adefovir add-on to lamivudine $(n=30)$, and adefovir-entecavir combination therapy $(n=32)$. The most significant decrease in HBV DNA levels from baseline was observed in patients receiving entecavir in combination with adefovir $\left(-5.58 \log _{10} \mathrm{IU} / \mathrm{mL}\right.$ at 24 months; $P<0.01$ versus other treatment groups). No incidences of viral breakthrough or genotypic resistance were observed in the combination-therapy group after 24 months $(P<0.05$ versus other treatment groups). Overall, the combination of entecavir and adefovir demonstrated faster and significantly greater suppression of HBV DNA levels compared with adefovir add-on to lamivudine. Therefore, as well as having a lower barrier to resistance as a monotherapy, entecavir can also be used as part of a combination therapy in patients refractory to lamivudine.

\section{Entecavir in adefovir-resistant CHB}

Adefovir-resistant mutations are also increasingly recognized. A number of studies have assessed the use of entecavir in adefovir-refractory Chinese patients with $\mathrm{CHB}$ (Table 2B). A prospective, controlled study compared the safety and efficacy of entecavir with adefovir in combination with telbivudine in patients with $\mathrm{CHB}$ who were resistant to adefovir. ${ }^{40}$ After 48 weeks of treatment, there were no statistically significant differences in rates of virologic response between the two treatment groups $(P=0.195)$. $\mathrm{HBe} A g$ loss was experienced by $33 \%$ of patients treated with combination therapy, compared with $11 \%$ of patients who received entecavir, and a similar rate of ALT normalization at week 48 was seen for both groups ( $85 \%$ for telbivudine and adefovir versus $71 \%$ for entecavir; $P=0.428$ ). Virologic breakthrough occurred in two entecavir-treated patients and one patient who received telbivudine plus adefovir, with no significant differences between the two 
Table 2 Summary of ETV efficacy either alone or in combination versus other nucleos(t)ide therapies in Chinese patients with drugresistant $\mathrm{CHB}^{\mathrm{a}}$

\begin{tabular}{|c|c|c|c|c|}
\hline & ADV monotherapy & ADV add-on to LVD & $A D V+E T V$ & ETV monotherapy \\
\hline \multicolumn{5}{|l|}{ (A) LVD-resistant CHB } \\
\hline \multicolumn{5}{|l|}{ Ha et $\mathrm{al}^{39}$} \\
\hline HBV DNA undetectable ${ }^{b}$ & 49 & 77 & 88 & NA \\
\hline ALT normalization ${ }^{c}$ & 72 & 80 & 88 & NA \\
\hline $\mathrm{HBeAg}$ loss $^{\mathrm{b}}$ & 35 & 50 & 53 & NA \\
\hline $\mathrm{HBeAg}$ seroconversion ${ }^{\mathrm{b}}$ & 21 & 30 & 38 & NA \\
\hline \multicolumn{5}{|l|}{ Zhao et $\mathrm{al}^{38}$} \\
\hline \multirow[t]{2}{*}{ ALT normalization ${ }^{c}$} & 75 & 84 & 100 & 93 \\
\hline & \multicolumn{3}{|c|}{ LdT add-on to ADV } & ETV monotherapy \\
\hline \multicolumn{5}{|l|}{ (B) ADV-resistant CHB } \\
\hline \multicolumn{5}{|l|}{ Lu et $\mathrm{al}^{40}$} \\
\hline HBV DNA undetectable ${ }^{d}$ & & 73 & & 57 \\
\hline ALT normalization ${ }^{d}$ & & 85 & & 71 \\
\hline $\mathrm{HBeAg}$ loss $^{d}$ & & 33 & & 11 \\
\hline \multirow[t]{2}{*}{$\mathrm{HBeAg}$ seroconversion ${ }^{d}$} & & 20 & & 0 \\
\hline & & \multicolumn{2}{|l|}{ LVD add-on to ADV } & ETV monotherapy \\
\hline \multicolumn{5}{|l|}{ Wang et al ${ }^{41}$} \\
\hline HBV DNA undetectable ${ }^{c}$ & & 97 & & 68 \\
\hline ALT normalization ${ }^{c}$ & & 97 & & 84 \\
\hline $\mathrm{HBeAg} \operatorname{loss}^{c}$ & & 47 & & 8 \\
\hline HBeAg seroconversion ${ }^{c}$ & & 37 & & 4 \\
\hline
\end{tabular}

Notes: All data are presented as percentage of patients, unless stated otherwise. a Direct comparisons cannot be made between studies due to differences in treatment periods and different experimental procedures between studies; ${ }^{\mathrm{b}} 24$ months of treatment; ${ }^{\mathrm{c}} / 2$ months of treatment; ${ }^{\mathrm{d}} 48$ weeks of treatment.

Abbreviations: ADV, adefovir; ALT, alanine transaminase; CHB, chronic hepatitis B; DNA, deoxyribonucleic acid; HBeAg, Hepatitis B e-antigen; HBV, hepatitis B virus; ETV, entecavir; LdT, tenofovir; LVD, lamivudine; NA, not applicable.

groups at week 48. Thus, entecavir demonstrated effective antiviral efficacy in patients who were resistant to adefovir. Another prospective, controlled study comparing entecavir monotherapy with lamivudine plus adefovir in adefovirrefractory Chinese patients with $\mathrm{CHB}$ also demonstrated favorable results with the use of entecavir. ${ }^{41}$ Biochemical response rates were $97 \%$ and $84 \%(P=0.097)$, and virologic response rates were $97 \%$ and $68 \%(P=0.003)$ in the lamivudine plus adefovir and entecavir monotherapy groups, respectively, demonstrating that single-agent entecavir is also an effective therapeutic strategy in adefovir-resistant Chinese patients with CHB.

\section{Entecavir in multidrug-resistant $\mathrm{CHB}$}

The superiority of add-on therapy has only recently been established, thus many patients with CHB have received sequential monotherapy regimens that have resulted in resistance to multiple therapies. Entecavir combination therapy may also be effective in these patients. The use of entecavir in combination with adefovir has been investigated in 52 Chinese patients with CHB who had failed two or more previous nucleos $(\mathrm{t})$ ide therapies. ${ }^{42}$ No patient treated with entecavir and adefovir combination therapy developed primary non-response or viral breakthrough during follow-up.
In addition, all patients achieved ALT normalization, and the rate of $\mathrm{HBeAg} /$ anti-HBe seroconversion was $16 \%$ (7/45 patients) at 12 months, and $27 \%$ (12/45 patients) at 24 months of treatment. No treatment-related adverse events were reported throughout the treatment period. Thus, entecavir in combination with adefovir can be considered an effective therapeutic regimen in patients with multiple nucleos $(\mathrm{t})$ ide therapy failures.

Entecavir has also been evaluated as both monotherapy and in combination with adefovir in Chinese patients with lamivudine-resistant CHB who have also failed prior lamivudine plus adefovir therapy. ${ }^{43}$ After 24 weeks of treatment, a significantly lower rate of HBV DNA levels $<500$ copies $/ \mathrm{mL}$ was seen in entecavir-treated patients than in those who received entecavir plus adefovir ( $29 \%$ versus $81 \%$; $P=0.004)$. By week 48, all patients in the entecavir-adefovir combination therapy group had achieved HBV DNA levels $<500$ copies/ $\mathrm{mL}$. ALT normalization was achieved by $43 \%$ and $92 \%$ of the entecavir and entecavir-adefovir combination therapy groups, respectively, at week 24 , and by $57 \%$ and $100 \%$, respectively, at week 48. Taken together, these studies demonstrate that the use of entecavir in combination with adefovir is associated with favorable antiviral efficacy in patients with multidrugresistant CHB. 


\section{Benefits of entecavir in Chinese patients with HBV- related conditions \\ Reduced risk of hepatic events}

HBV infection has consistently been demonstrated to be an independent risk factor for development of cirrhosis and hepatocellular carcinoma. ${ }^{44}$ As such, the main goal of therapy for $\mathrm{CHB}$ is to prevent the development of long-term complications of HBV infection, including cirrhosis and hepatocellular carcinoma. ${ }^{3}$

A retrospective cohort study from Hong Kong examined the efficacy of entecavir with regard to clinical outcomes and death rates, with the primary outcome of a 5 -year cumulative probability of hepatic events, defined as any cirrhotic complication, hepatocellular carcinoma, or liver-related mortality. ${ }^{44}$ In 482 patients with cirrhosis who received entecavir therapy, there was a reduced risk of all clinical outcomes compared with a cohort of treatment-naïve patients with cirrhosis. However, in entecavir-treated patients who failed to achieve undetectable HBV DNA levels, the risk of developing a hepatic complication was comparable with that in untreated patients. The authors concluded that entecavir reduces the risk of hepatic events, particularly among individuals with maintained viral suppression.

The beneficial effects of a virologic response to entecavir in preventing progression of liver disease in patients with and without cirrhosis have also been observed in a study from Taiwan. ${ }^{45}$ Further, a virologic response to entecavir reduced the risk of clinical events and hepatocellular carcinoma in nucleos $(\mathrm{t})$ ide-experienced patients with prior lamivudine-resistant or adefovir-resistant mutants, but not in nucleos $(\mathrm{t})$ ide-naïve patients or nucleos $(\mathrm{t})$ ide-experienced patients who had never developed lamivudine-resistant or adefovir-resistant mutants.

A real-world study in Taiwan investigated the safety and efficacy of entecavir in treatment-naïve, $\mathrm{HBeAg}$-positive patients with compensated liver disease. ${ }^{46}$ Long-term entecavir therapy was associated with favorable biochemical and virologic responses, but only modest serologic responses. Baseline ALT levels more than five times the upper limit of normal and lower baseline HBV DNA levels were also demonstrated to be favorable predictors of serologic response.

\section{Efficacy in patients}

\section{with decompensated cirrhosis}

More recently, a growing body of data has demonstrated that entecavir is associated with favorable safety and efficacy in
CHB patients with decompensated cirrhosis. ${ }^{47}$ Liaw et al reported that entecavir $1 \mathrm{mg}$ /day was associated with superior antiviral efficacy at week 48 compared with adefovir $10 \mathrm{mg}$ /day (the proportion of patients achieving HBV DNA $<300$ copies $/ \mathrm{mL}$ was $57 \%$ versus $20 \%$ respectively; $P<0.0001)$ in patients with CHB who had hepatic decompensation (Child-Turcotte-Pugh score $\geq 7$ ). ${ }^{48}$ Approximately two thirds of patients in both treatment groups showed stabilization or improvement in Child-Turcotte-Pugh scores. The model for end-stage liver disease score at week 48 was also improved in both treatment groups; the score for entecavir was 22.6 (baseline 17.1) and for adefovir was 21.7 (baseline 15.3). Adverse event rates were comparable between the groups, and cumulative hepatocellular carcinoma rates were $12 \%$ for entecavir patients and $20 \%$ in patients treated with adefovir. In another study, reported by Liaw et al, entecavir was well tolerated in patients with decompensated liver disease, with an overall improvement in virologic, biochemical, and clinical parameters being demonstrated. ${ }^{49}$ However, long-term studies in Chinese patients with CHBassociated decompensated cirrhosis are required to confirm these findings.

\section{Efficacy in patients with HBV-associated liver failure}

CHB is the most common cause of liver failure, and can develop as acute liver failure, acute-on-chronic liver failure, or chronic decompensation of end-stage liver disease. Although the US guidelines for diagnosis and treatment of CHB recommend entecavir as a first-line antiviral drug for patients with HBV-related liver failure, ${ }^{50}$ data are lacking to support the use of entecavir in Chinese patients.

A recent meta-analysis demonstrated that nucleos(t)ide analog therapy, including entecavir, was associated with improved survival, significant reductions in serum HBV DNA levels at 3 months, and significantly higher rates of $\mathrm{HBeAg}$ seroconversion..$^{51}$ These findings are in agreement with those of Cui et al, who observed that although entecavir or lamivudine did not significantly increase 3-month survival rates in patients with HBV-associated acute-onchronic liver failure compared with patients who received no therapy, treatment with either nucleos(t)ide analog was associated with significantly lower levels of HBV DNA and lower rates of recurrence of acute-on-chronic liver failure. ${ }^{52}$ Further, timely administration of entecavir has been associated with an improved prognosis in patients with $\mathrm{HBe} A g$-negative acute-on-chronic liver failure. ${ }^{53}$ Further research is required to assess the therapeutic use 
of entecavir in Chinese patients with liver failure attributed to $\mathrm{CHB}$ infection.

\section{Benefits of entecavir treatment after liver transplantation Prevention of recurrence of $\mathrm{HBV}$}

Liver transplantation is the only treatment strategy available for HBV-related end-stage liver disease. ${ }^{54}$ However, HBV recurrence is a frequent complication, occurring in up to $12 \%$ of patients. ${ }^{54}$ Although the use of hepatitis B immunoglobulin plus lamivudine has reduced the HBV recurrence rate, long-term use of lamivudine is associated with a high rate of resistance in $\mathrm{HBV}$, as are other nucleotide analogs, such as adefovir and telbivudine. Entecavir is associated with a lower barrier to resistance, so may be an effective strategy in preventing $\mathrm{HBV}$ recurrence in patients undergoing liver transplantation.

A number of studies have demonstrated that entecavir alone or in combination with HBV immunoglobulin can prevent recurrence of $\mathrm{HBV}$ after liver transplantation. In one small-scale study, the combination of entecavir with low-dose HBV immunoglobulin was shown to be an effective and well tolerated strategy for preventing HBV recurrence after liver transplantation. ${ }^{54} \mathrm{~A}$ retrospective case-controlled study, which enrolled 252 patients between 2005 and 2007, evaluated the effects of entecavir plus lamivudine in preventing HBV recurrence after orthotopic liver allograft in Chinese patients with $\mathrm{HBV}$-related end-stage liver disease. ${ }^{55}$ A total of 18 patients who received lamivudine developed HBV reinfection, with an $\mathrm{HBV}$ reinfection rate of $10 \%$. In contrast, no patients in the entecavir group experienced reinfection. The difference in reinfection rates between the two treatment groups was statistically significant $(P<0.01)$, as was the difference in cumulative reinfection rate $(P<0.01)$.

These findings were confirmed by Fung et al who examined the efficacy of entecavir monotherapy in suppressing HBV in Chinese patients with HBV who underwent liver transplantation. ${ }^{56}$ At the time of transplantation, $26 \%$ of patients had complete viral suppression. The cumulative rate of HBsAg loss was $86 \%$ and $91 \%$ after 1 and 2 years of entecavir treatment, respectively. This study demonstrated that an HBV immunoglobulin-free regimen of entecavir monotherapy is effective for suppressing HBV in individuals undergoing liver transplantation.

A study in patients undergoing liver transplantation in Hong Kong also investigated the use of lamivudine, entecavir, or lamivudine-entecavir combination therapy. ${ }^{57}$ The rates of undetectable HBV DNA at 1, 3, 5, and 8 years were $94 \%, 96 \%, 96 \%$, and $98 \%$, respectively, using oral antiviral therapy alone. This study demonstrated that oral nucleos(t)ide prophylaxis without HBV immunoglobulin is associated with excellent long-term survival in patients with CHB after liver transplantation. For those without pre-existing drug-resistant mutations, using an antiviral agent with a high barrier to resistance, such as entecavir, is recommended. Combination therapy is recommended for those with pre-existing drug-resistant mutations.

\section{Treatment of immunosuppressed individuals}

Patients with active HBV infection and those who have previously been infected with HBV but have apparently cleared the virus (HBsAg-negative, but with antibodies against hepatitis $\mathrm{B}$ core or surface antigen) and undergo immunosuppressive therapy are at risk of $\mathrm{HBV}$ reactivation. ${ }^{58}$ Reactivation is associated with a number of poor clinical outcomes, including elevation of serum HBV DNA and/or serum transaminase levels, jaundice, fulminant hepatic failure, and liver-related death. ${ }^{59}$ Use of anti-HBV therapies during immunosuppressive therapy can prevent $\mathrm{HBV}$ reactivation. A recent study compared the efficacy of entecavir with that of lamivudine for preventing HBV reactivation in patients with lymphoma who were receiving chemotherapy. ${ }^{60}$ The entecavir-treated group had significantly lower rates of hepatitis ( $6 \%$ versus $27 \% ; P=0.007)$, HBV reactivation ( $0 \%$ versus $12 \% ; P=0.024)$, and disruption of chemotherapy ( $6 \%$ versus $20 \% ; P=0.042$ ), demonstrating that entecavir is more effective than lamivudine in the prevention of $\mathrm{HBV}$ reactivation in patients receiving chemotherapy.

\section{Summary}

Overall, entecavir has demonstrated effective antiviral suppression and good tolerability in Chinese patients with CHB. In this patient population, entecavir has shown antiviral efficacy comparable with that of other recommended nucleos(t)ide therapies, including lamivudine and adefovir, with a number of studies demonstrating superior efficacy with entecavir. It has also shown efficacy in lamivudine-resistant patients, either alone or in combination with other nucleos(t)ides. Entecavir reduces the risk of hepatic events in Chinese patients with $\mathrm{CHB}$, and has demonstrated efficacy in those with HBV-associated decompensated cirrhosis and liver failure. In addition, entecavir can prevent $\mathrm{HBV}$ reactivation in patients undergoing immunosuppressive therapy. 


\section{Challenges and strategies with use of entecavir in the People's Republic of China}

Despite its demonstrable efficacy, a number of challenges remain regarding entecavir therapy in the People's Republic of China. None of the currently available therapies for $\mathrm{CHB}$, including entecavir, induce HBsAg loss or seroconversion in the majority of patients who receive treatment. Strategies required to overcome this challenge include the exploration of combination treatment using entecavir and pegIFN- $\alpha^{61}$ or the use of therapeutic vaccines. ${ }^{62}$ In addition, because HBV infection cannot be cured, there is a high rate of HBV reactivation. In patients who experience recurrence, therapy cannot be stopped, and it is important to restore immune responses in patients in whom reactivation occurs. Therefore, the addition of IFN therapy may be an important consideration, although further research is needed to confirm this.

Another major challenge is that physicians in the People's Republic of China, particularly those in gastroenterology, geriatrics, and other hospital departments, demonstrate a lack of awareness of CHB guidelines, especially those working in hospitals not affiliated with a university. ${ }^{4}$ This may result in patients not receiving the currently recommended first-line anti-HBV therapies, which may lead to a poorer prognosis for some. Improved education concerning the management of HBV infection is required, particularly for physicians based in county hospitals or clinics.

Medical insurance and payment capability regarding CHB treatment is also a challenge for Chinese patients with CHB. Although there is a short-term study demonstrating similar efficacy between entecavir (Baraclude) and less expensive, generic entecavir, ${ }^{11}$ there is a lack of long-term evidence regarding generic entecavir drugs, so long-term follow-up studies are needed to support their use. In addition, policymakers need to objectively evaluate the optimization of treatment regimens by considering the findings of pharmacoeconomic studies that have recently demonstrated the cost-effective nature of entecavir compared with other nucleos(t)ide therapies.

Studies in the People's Republic of China have shown that entecavir has an efficacy profile in Chinese patients with CHB comparable with that observed in other ethnic patient populations. However, further research is needed to assess the use of entecavir combinations in the treatment of lamivudine-resistant disease, and in increasing HBsAg seroconversion rates in different patient populations. Improved education on the use of entecavir, particularly regarding its long-term effectiveness, is also required to ensure that both patients and physicians are aware of the clinical use of this anti-HBV therapy.

\section{Disclosure}

JW has no conflict of interests to declare in relation to this review. Preparation of the manuscript was assisted by ArticulateScience (UK), which is contracted by BristolMyers Squibb.

\section{References}

1. Zhang C, Zhong Y, Guo L. Strategies to prevent hepatitis B virus infection in China: immunization, screening, and standard medical practices. Biosci Trends. 2013;7:7-12.

2. Chinese Society of Hepatology and Chinese Society of Infectious Diseases, Chinese Medical Association. [The guideline of prevention and treatment for chronic hepatitis B (2010 version)]. Zhonghua Gan Zang Bing Za Zhi. 2011;19:13-24. Chinese.

3. Liaw YF, Leung N, Kao JH, et al. Asian-Pacific consensus statement on the management of chronic hepatitis B: a 2012 update. Hepatol Int. 2012;6:263-283.

4. Ning LH, Hao J, Liao ZL, Zhou YY, Guo H, Zhao XY. A survey on the current trends in the management of hepatitis B in China. Eur $J$ Gastroenterol Hepatol. 2012;24:884-889.

5. Sun J, Hou JL. Management of chronic hepatitis B: experience from China. JViral Hepat. 2010;17 Suppl 1:10-17.

6. Anonymous. IMS China Hospital Market Overview (CHPA, 2012). 2012.

7. Yang ZG, Li YF, Zhang L, Wang J. A comparative study of entecavir dispersible tablets with entecavir in chronic hepatitis B. Journal of Bethune Military Medical College. 2012;10:110-111.

8. Yang GY, Zhang YG, Chen K, Chen J, Zhu YJ, Wang DW. Initial treatment of entecavir dispersible tablets in chronic hepatitis B: analysis of 52 cases. Chinese Medicine Guide. 2011;9:371-373.

9. Lv C, Zhang MQ. Efficacy of entecavir dispersible tablets in HBeAgpositive chronic hepatitis B patients. Journal of Medical Theory and Practice. 2012;25:663-664.

10. Xie YM, Zhu JR, Zhou WG. Cost-effectiveness analysis of domestic and imported entecavir in chronic hepatitis B. Journal of Medical Theory and Practice. 2012;25:2438-2439.

11. $\mathrm{Xu} \mathrm{JH,} \mathrm{Yu} \mathrm{YY,} \mathrm{Si} \mathrm{CW,} \mathrm{et} \mathrm{al.} \mathrm{[A} \mathrm{randomized,} \mathrm{double-blind,} \mathrm{double-}$ dummy, controlled, multicenter study of entecavir maleate versus entecavir for treatment of HBeAg-negative chronic hepatitis B: results at week 48]. Zhonghua Gan Zang Bing Za Zhi. 2012;20:512-516. Chinese.

12. Luo J, Li X, Wu Y, et al. Efficacy of entecavir treatment for up to 5 years in nucleos(t)ide-naive chronic hepatitis B patients in real life. Int J Med Sci. 2013;10:427-433.

13. Yuen MF, Seto WK, Fung J, Wong DK, Yuen JC, Lai CL. Three years of continuous entecavir therapy in treatment-naive chronic hepatitis B patients: viral suppression, viral resistance, and clinical safety. Am J Gastroenterol. 2011;106:1264-1271.

14. Chen J, Han JH, Liu C, et al. Short-term entecavir therapy of chronic severe hepatitis B. Hepatobiliary Pancreat Dis Int. 2009;8: 261-266.

15. Huang LH, Qiu YW, Hua HY, et al. The efficacy and safety of entecavir in patients with advanced schistosomiasis co-infected with hepatitis B virus. Int J Infect Dis. 2013;17:e606-e609.

16. Madwar MA, el Tahawy M, Strickland GT. The relationship between uncomplicated schistosomiasis and hepatitis B infection. Trans $R$ Soc Trop Med Hyg. 1989;83:233-236.

17. Wei L, Hu S, Hou J, et al. A novel estimation of the impact of treatment with entecavir on long-term mortality, morbidity, and health care costs of chronic hepatitis B in China. Value in Health Regional Issues. 2013;2:48-56.

18. Jin X, Chen YP, Yang YD, Li YM, Zheng L, Xu CQ. Association between hepatic steatosis and entecavir treatment failure in Chinese patients with chronic hepatitis B. PLoS One. 2012;7:e34198. 
19. Yao G. Entecavir is a potent anti-HBV drug superior to lamivudine: experience from clinical trials in China. J Antimicrob Chemother. 2007;60:201-205.

20. Hou J, Jia J, Wei L, et al. Entecavir versus other standard of care for up to 4 years in nucleos(t)ide-naive patients with $\mathrm{CHB}$ in Chinese clinical practice. Abstract presented at the Third Asian Pacific Association for Study of the Liver, June 6-10, 2013, Singapore.

21. Yao GB, Ren H, Xu DZ, et al. Virological, serological and biochemical outcomes through 3 years of entecavir treatment in nucleoside-naive Chinese chronic hepatitis B patients. J Viral Hepat. 2010;17 Suppl 1: 51-58.

22. Yao G, Chen C, Lu W, et al. Efficacy and safety of entecavir compared to lamivudine in nucleoside-naive patients with chronic hepatitis B: a randomized double-blind trial in China. Hepatol Int. 2007;1: 365-372.

23. Ren FY, Piao DM, Piao XX. A one-year trial of entecavir treatment in patients with $\mathrm{HBeAg}$-positive chronic hepatitis B. World $J$ Gastroenterol. 2007;13:4264-4267.

24. Yao G, Chen C, Lu W, et al. Virologic, serologic, and biochemical outcomes through 2 years of treatment with entecavir and lamivudine in nucleoside-naive Chinese patients with chronic hepatitis B: a randomized, multicenter study. Hepatol Int. 2008;2:486-493.

25. Chen EQ, Zhou TY, Liu L, et al. A comparison of treatment with adefovir and entecavir for chronic hepatitis B in China: the 2-year results of a prospective study: adefovir versus entercavir for chronic hepatitis B. Hepat Mon. 2011;11:27-31.

26. Wang LC, Chen EQ, Cao J, et al. De novo combination of lamivudine and adefovir versus entecavir monotherapy for the treatment of naive $\mathrm{HBe} A g$-negative chronic hepatitis B patients. Hepatol Int 2011;5:671-676.

27. Yan J, Ren N, Cai H, et al. The comparison of 96 weeks efficacy of lamivudine plus adefovir de novo combination therapy and entecavir monotherapy for HBeAg-positive. Hepatol Int (2013) 7 (Suppl 1):S1-S754.

28. Zheng MH, Shi KQ, Dai ZJ, Ye C, Chen YP. A 24-week, parallel-group, open-label, randomized clinical trial comparing the early antiviral efficacy of telbivudine and entecavir in the treatment of hepatitis B e antigen-positive chronic hepatitis B virus infection in adult Chinese patients. Clin Ther. 2010;32:649-658.

29. Tsai MC, Chen $\mathrm{CH}$, Hung $\mathrm{CH}$, et al. A comparison of efficacy and safety of 2-year telbivudine and entecavir treatment in patients with chronic hepatitis B: a match-control study. Clin Microbiol Infect. May 10, 2013. [Epub ahead of print.]

30. Wu B, Li T, Chen H, Shen J. Cost-effectiveness of nucleoside analog therapy for hepatitis B in China: a Markov analysis. Value Health. 2010;13:592-600.

31. Lee KK, Wu DB, Chow PY, Lee VW, Li H. Economic analysis between entecavir and lamivudine for the treatment of chronic hepatitis B in Hong Kong. J Gastroenterol Hepatol. 2012;27: 1167-1174.

32. Lau DT, Khokhar MF, Doo E, et al. Long-term therapy of chronic hepatitis B with lamivudine. Hepatology. 2000;32:828-834.

33. Liaw YF. Impact of YMDD mutations during lamivudine therapy in patients with chronic hepatitis B. Antivir Chem Chemother. 2001; 12 Suppl 1:67-71

34. Ono SK, Kato N, Shiratori Y, et al. The polymerase L528M mutation cooperates with nucleotide binding-site mutations, increasing hepatitis B virus replication and drug resistance. J Clin Invest. 2001;107: 449-455.

35. Zoulim F. Detection of hepatitis B virus resistance to antivirals. J Clin Virol. 2001;21:243-253.

36. Liu Y, Wang C, Zhong Y, et al. Genotypic resistance profile of hepatitis B virus (HBV) in a large cohort of nucleos(t)ide analogueexperienced Chinese patients with chronic HBV infection. J Viral Hepat. 2011;18:e29-e39.

37. Yao G, Zhou X, Xu D, et al. Entecavir for the treatment of lamivudinerefractory chronic hepatitis B patients in China. Hepatol Int. 2007;1: 373-381.
38. Zhao P, Wang C, Huang L, Xu D, Li T. Comparison of rescue strategies in lamivudine-resistant patients with chronic hepatitis B. Antiviral Res. 2012;96:100-104.

39. Ha M, Zhang G, Diao S, et al. Rescue therapy for lamivudine-resistant chronic hepatitis B: adefovir monotherapy, adefovir plus lamivudine or entecavir combination therapy. Intern Med. 2012;51:1509-1515.

40. Lu JJ, Liu K, Ma YJ, Wang J, Chen E-Q, Tang H. Efficacy and safety of telbivudine plus adefovir dipivoxil combination therapy and entecavir monotherapy for HBeAg-positive chronic hepatitis B patients with resistance to adefovir dipivoxil. J Viral Hepat. 2013;20 Suppl 1: $40-45$.

41. Wang JC, He LL, Chen Q. Comparison of re-treatment outcomes of lamivudine plus adefovir or entecavir in chronic hepatitis B patients with viral relapse after cessation of adefovir. Eur Rev Med Pharmacol Sci. 2013;17:1162-1166.

42. Xu XH, Li GL, Qin Y, et al. Entecavir plus adefovir rescue therapy for chronic hepatitis B patients after multiple treatment failures in real-life practice. Virol J. 2013;10:162.

43. Xing J, Han T, Liu L, et al. [Entecavir $1.0 \mathrm{mg}$ monotherapy or entecavir plus adefovir dipivoxil for patients with lamivudine-resistant chronic hepatitis B had suboptimal response to lamivudine plus adefovir dipivoxil]. Zhonghua Gan Zang Bing Za Zhi. 2011;19:828-832. Chinese.

44. Wong GL, Chan HL, Mak CH, et al. Entecavir treatment reduces hepatic events and deaths in chronic hepatitis B patients with liver cirrhosis. Hepatology. February 6, 2013. [Epub ahead of print.]

45. Yang SC, Lee CM, Hu TH, et al. Virological response to entecavir reduces the risk of liver disease progression in nucleos $(\mathrm{t})$ ide analogueexperienced HBV-infected patients with prior resistant mutants. J Antimicrob Chemother. 2013;68:2154-2163.

46. Wang CC, Tseng KC, Peng CY, et al. Viral load and alanine aminotransferase correlate with serologic response in chronic hepatitis B patients treated with entecavir. J Gastroenterol Hepatol. 2013;28: 46-50.

47. Basu PP, Brown RS Jr. Entecavir for the treatment of chronic hepatitis B: A clinical update for the treatment of patients with decompensated cirrhosis. Open J Intern Med. 2012;2:53-61.

48. Liaw YF, Raptopoulou-Gigi M, Cheinquer H, et al. Efficacy and safety of entecavir versus adefovir in chronic hepatitis B patients with evidence of hepatic decompensation. Hepatology. 2011;54:91-100.

49. Liaw YF, Sheen IS, Lee CM, et al. Tenofovir disoproxil fumarate (TDF), emtricitabine/TDF, and entecavir in patients with decompensated chronic hepatitis B liver disease. Hepatology. 2011;53:62-72.

50. Lok AS, McMahon BJ. Practice Guidelines Committee, American Association for the Study of Liver Diseases (AASLD). Chronic hepatitis B: update of recommendations. Hepatology. 2004;39:857-861.

51. Xie F, Yan L, Lu J, et al. Effects of nucleoside analogue on patients with chronic hepatitis B-associated liver failure: meta-analysis. PLoS One. 2013;8:e54773.

52. Cui YL, Yan F, Wang YB, et al. Nucleoside analogue can improve the longterm prognosis of patients with hepatitis B virus infection-associated acute on chronic liver failure. Dig Dis Sci. 2010;55:2373-2380.

53. Yan Y, Mai L, Zheng YB, et al. What MELD score mandates use of entecavir for ACLF-HBV HBeAg-negative patients? World $J$ Gastroenterol. 2012;18:4604-4609.

54. Xie SB, Zhu JY, Ying Z, Zeng LJ, Chao M, Lu MQ. Prevention and risk factors of the HBV recurrence after orthotopic liver transplantation: 160 cases follow-up study. Transplantation. 2010;90:786-790.

55. Cai CJ, Lu MQ, Chen YH, Zhao H, Li MR, Chen GH. Clinical study on prevention of HBV re-infection by entecavir after liver transplantation. Clin Transplant. 2012;26:208-215.

56. Fung J, Cheung C, Chan SC, et al. Entecavir monotherapy is effective in suppressing hepatitis B virus after liver transplantation. Gastroenterology. 2011;141:1212-1219.

57. Fung J, Chan SC, Cheung C, et al. Oral nucleoside/nucleotide analogs without hepatitis B immune globulin after liver transplantation for hepatitis B. Am J Gastroenterol. 2013;108:942-948. 
58. Liu CJ, Chen PJ, Chen DS, Kao JH. Hepatitis B virus reactivation in patients receiving cancer chemotherapy: natural history, pathogenesis, and management. Hepatol Int. June 14, 2011. [Epub ahead of print.]

59. Liang RH, Lok AS, Lai CL, Chan TK, Todd D, Chiu EK. Hepatitis B infection in patients with lymphomas. Hematol Oncol. 1990;8:261-270.

60. Li HR, Huang JJ, Guo HQ, et al. Comparison of entecavir and lamivudine in preventing hepatitis B reactivation in lymphoma patients during chemotherapy. J Viral Hepat. 2011;18:877-883.
61. Chen X, Cao Z, Liu Y, et al. Potent hepatitis B surface antigen response to treatment of hepatitis-B-e-antigen-positive chronic hepatitis B with alpha-interferon plus a nucleos(t)ide analog. J Gastroenterol Hepatol. 2012;27:481-486.

62. Xu DZ, Wang XY, Shen XL, et al. Results of a Phase III clinical trial with an HBsAg-HBIG immunogenic complex therapeutic vaccine for chronic hepatitis B patients: experiences and findings. $J$ Hepatol. 2013;9:450-456

\section{Publish your work in this journal}

Drug Design, Development and Therapy is an international, peerreviewed open-access journal that spans the spectrum of drug design and development through to clinical applications. Clinical outcomes, patient safety, and programs for the development and effective, safe, and sustained use of medicines are a feature of the journal, which has also been accepted for indexing on PubMed Central. The manuscript management system is completely online and includes a very quick and fair peer-review system, which is all easy to use. Visit http://www.dovepress.com/testimonials.php to read real quotes from published authors.

Submit your manuscript here: http://www.dovepress.com/drug-design-development-and-therapy-journal 